clozapine?) or does he regularly show 'simple entertainment' videos to families?

I am not surprised that many of the families 'love it'; presumably when it confirms improvement and affirms hope for the future. What of those for whom clozapine has proved less successful?

I, too, welcome hope for people with schizophrenia and their families and recognise the important contribution of clozapine. I am not sure that pointing out one or two biases and a dislike of sentimentality need totally dismiss hope and wonder at the sensitivity of those who see them as such.

Glasgow University Media Group (1993a) Mass media representations of mental health/illness. Report for the Health Education Board for Scotland.

- (1993b) Media representations of mental health/ulness: audience reception study. Report for the Health Education Board for Scotland.

Jacgueline M. Atkinson, Department of Public Health, University of Glasgow, Glasgow G12 8RZ

\section{Routine blood monitoring in epileptic patients with learning disability}

Sir: The audit of Amaladoss \& Arumainayagam concerned epilepsy in this predisposed and vulnerable group (Psychiatric Bulletin. 1994, 18, 680-682). Their assertion that there is little justification for routine annual blood monitoring for epileptic patients is questionable, especially as only 75 cases were surveyed. Most clinicians are familiar with the argument that routine blood monitoring may identify unsuspected subtherapeutic or toxic drug levels in a patient with major communication difficulties. Furthermore, although routine measurements will not predict acute idiosyncratic drug reactions, subclinical biochemical or haematological deterioration may be discovered. This is especially important with valproate-associated hepatotoxicity-hepatic enzymes may be raised on routine monitoring when the hepatotoxicity can be reversed but this is unlikely to be the case by the time overt clinical signs develop. Careful clinical judgement is required to decide when to reduce or withdraw valproate in the light of borderline abnormalities (Wyllie \& Wyllie, 1991). In one series, 36 of 37 cases of fatal valproate-associated hepatic failure had learning disability, developmental delay or congenital anomalies (Dreifuss et al, 1987). Therefore, although such fatalities are rare, we should be particularly vigilant in the learning disability population - especially as those with a lesional basis for their difficult to control seizures may well receive multiple antiepileptic drugs which is itself a risk factor for drugassociated hepatotoxicity. In this regard, it is debatable whether best clinical practice can be defined using small audit projects aimed at measuring current practice rather than clinical research per se. Similar considerations suggest that routine haematological monitoring may identify clinically unsuspected cases of significant valproateinduced thrombocytopenia as well as chronic leucopenia associated with carbamazepine or ethosuximide.

Routine blood monitoring also provides an opportunity to ensure that other appropriate tests are done, for example, thyroid function and lithium levels, in a group of patients whose access to primary care services is at times tenuous. We are currently undertaking an audit of epilepsy care in learning disability in the Oxford region. Participating consultants expressed a variety of views on the need for routine blood monitoring but the consensus opinion was in favour of annual measures of: antiepileptic drug levels, routine biochemistry and haematology and folate levels in the few taking phenytoin or phenobarbitone.

DREIFUSS, F. E., SANTILU, N., LANGER, D. et al (1987) Valproic acid hepatic fatalities a retrospective review. Neurology. 37. 379-385.

WYLLE, E. \& WYLLE, R. (1991) routine laboratory monitoring for serious adverse effects of antiepileptic medications: the controversy. Epilepsia, 32(Suppl.5), S74-S79.

D. Rowe, Princess Marina Hospital, Upton, Northampton NN5 4UN and RACHEL JAMES, Four Counties Clinical Audit Team, Old Road, Headington, Oxford OX3 7LF

Sir: Amaladoss \& Arumainayagam's audit concerned epilepsy in an in-patient mentally handicapped population (Psychiatric Bulletin, 1994, 18, 680-682). We conducted a similar audit on 154 in-patients within Phoenix Trust in June 1992. We do not agree with Amaladoss \& Arumainayagam that there is little justification for any annual blood monitoring in the mentally handicapped who are on anticonvulsant medication. 
We were puzzled by their rationale for routine serum investigations, in particular the annual measurement of folate levels, thyroid function. serum calcium, alkaline phosphatase and full blood counts in all patients irrespective of the anticonvulsant medication prescribed. We would suggest that serological monitoring is tailored for the drugs prescribed. Serum folate levels, for example, only need measuring if prescribing phenytoin and should be done six monthly as advised by the data sheet. Thyroid function and serum calcium are not recommended investigations for any anticonvulsant as far as we are aware. Only carbamazepine frequently induces hyponatraemia and justifies electrolytes being measured on a six monthly basis.

In our audit we found significant clinical and biochemical side-effects from anticonvulsant medication which affected drug therapy. Twenty-eight patients on carbamazepine had an identifiable abnormality: $8 \%$ with low sodium, $1 \%$ with high sodium, $3 \%$ with low potassium, 4\% with other electrolyte abnormalities and $2 \%$ with abnormal full blood counts. In addition $11 \%$ had a raised alkaline phosphatase and $11 \%$ had high drug levels. Although neither of these latter states in themselves required intervention, it was felt to be useful for the clinician to be aware of this. We also identified 13 abnormal results in patients on sodium valproate which included five (14\%) patients with high alkaline phosphatase, one $(3 \%)$ with an abnormal full blood count, four (11\%) with other electrolyte abnormalities and five (14\%) with low sodium (three of these patients were also on carbamazepine). Several of these results triggered clinical review of therapy.

We suggest that the tailored monitoring of haematological, biochemical indices and some anticonvulsant levels is an important part of day to day monitoring of the effects of epilepsy and that failure to do so may cause patients to suffer and leave little justification in the court room especially given the data sheet recommendations.

Clare Short and Peter Carpenter, Stoke Park Hospital, Stapleton, Bristol BS16 1QU

\section{Clozapine prescribing}

Sir: We read with interest the survey on clozapine prescribing undertaken by Seabourne \& Thomas (Psychiatric Bulletin. $1994,18,618-619)$.
A similar audit was recently completed by ourselves. Comparable findings included age, diagnosis and chronicity of illness. Doses of clozapine were similar (mean $=385 \mathrm{mg} /$ day) as were reasons for discontinuing treatment; although in our sample $16 / 19(63 \%)$ have received clozapine without interruption for at least 18 months, while only three deregistered permanently (all within five months of starting the drug).

Most patients had positive symptoms of schizophrenia (85\%). In addition, a relattvely high proportion exhibited secondary mood $(40 \%)$, or behavioural disturbance $(50 \%)$. Definite improvement, in at least some aspects of mental state, was recorded in 11/16 (68\%), with, in particular, an improvement in posittive symtomatology (delusions and hallucinations). As perhaps expected, clozapine was less beneficial for those patients with mood impairment, or aggressive or overactive behaviour. In two patients, no change was recorded.

In common with Seabourne \& Thomas, we found that accurate documentation of mental state and recording of change over time, was lacking. The response to clozapine of six patients $(27 \%)$ could not be ascertained by inspection of the case-notes. Similarly, dates of commencement on clozapine, current dose and reasons for starting (or discontinuing) the drug, were not always clear, or easy to find.

Measures to improve and objectify recording of patients' response to treatment would benefit patient care, help justify the use of more costly drugs and facilitate future audit. We suggest a 'clozapine front sheet', to be inserted in the notes, giving easy access to basic information such as start date, dose changes, and progressive and objective ratings of mental state. This could also include an indication that the patient fulfils locally agreed prescribing criteria. Confirmation that full information had been given to the patient would help to signify their commitment to the blood testing regime and ensure that risks of blood dyscrasia etc had been discussed.

Regular reviews in a dedicated clozapine clinic' rather than in general psychiatry outpatient clinics could ensure that this information is collected in a systematic and comprehensive manner.

Jonathan C. Hillam, Susan Shaw and Amanda Thompsell, Felix Brown Day Unit, Royal Free Hospital, London NW3 